\title{
LA MATERNIDAD TARDÍA: Expresión contemporánea del patriarcado occidental
}

Ma Cándida ALAMILLOS GUARDIOLA Universidad de las Islas Baleares mc.alamillos@uib.es, macalallos@gmail.com

\section{LATE MATERNITY: Contemporary Expression of Western Patriarchy}

Resumen: En las sociedades europeas occidentales se ha producido un aumento de las tasas de infertilidad. Una de las causas es el incremento en la edad de la pareja. La mujer se enfrenta a la maternidad de forma planificada retrasando el proceso en el tiempo en pro de estabilidad económica y profesional. Es la maternidad tardía, cuyo análisis parte de la construcción sociocultural del rol femenino de esposa/madre dentro de la estructura patriarcal tradicional.

Abstract: In western European societies there was an increment of infertility. The age of the couple is one of the reason. Women go to maternity later, planifiying it, looking forward the best laboral and economic situation. From social and cultural women's construction of mother and wife roles inside of patriarchal structure, maternity as a concept is made from men's point of view. Nowadays western women have late maternity, that it will be studied in this paper.

Palabras clave: Infertilidad. Patriarcado. Maternidad

Infertility. Patriarchy. Maternity 


\section{Introducción}

Las mujeres de Europa occidental afrontan el siglo XXI desde una perspectiva inédita en la historia, construyendo un espacio propio femenino dentro de un ámbito público estructurado desde el dominio masculino. La incorporación de la mujer al mundo profesional conlleva una readaptación del mundo privado. El binomio matrimonio/maternidad está cambiando. El perfil de esposa deriva hacia una mujer independiente y trabajadora con las mismas obligaciones domesticas que el esposo. La maternidad evoluciona, mientras los medios de comunicación difunden noticias en relación a un descenso de la natalidad/fertilidad en toda Europa. Son varias las causas de infertilidad, pero se adjudica gran importancia al incremento de la edad de los progenitores. La edad media de la mujer en España para su primer embarazo es de 32,1 años, mientras que en 1990 era de 28,8 años. La tasa de fertilidad de una mujer de 32 años es del 100\%. A partir de esta edad se produce un descenso progresivo de la misma, alcanzando a los 41 años un valor de 20\%. El tiempo "fértil" de la mujer está disminuyendo al optar más tarde al embarazo, sin que las condiciones biológicas se hayan modificado. Actualmente ambos miembros de la pareja deciden tener hijos más tarde, decisión influenciada por las características de las sociedades occidentales en las que prevalecen estilos de vida que posponen la maternidad/paternidad en beneficio de otros objetivos. Mientras que en épocas tradicionales el matrimonio y los hijos eran el fin fundamental para cualquier mujer, hoy en día estas opciones son relegadas a un futuro, dando preferencia a la formación y estabilidad profesional (Delgado 1993:123-154).

El análisis de la maternidad tardía debe partir de la situación de la mujer en el patriarcado, considerándolo como una herramienta de cohesión social tradicional que perpetuó la concepción de la mujer como madre y esposa. También se debe tener en cuenta la evolución del concepto de maternidad como construcción cultural en la postmodernidad.

\section{Patriarcado}

Sin profundizar en exceso en las concepciones filosóficas que adjudican a la mujer un estado de inferioridad naturalizada frente al varón, la construcción sociocultural se realiza en base al dimorfismo sexual propio de nuestra especie. Desde una perspectiva masculina se establece lo que Bourdieu describe como "trabajo colectivo de socialización de lo biológico y de biologización de lo social". Se naturaliza la conceptualización de la diferencia de género, cuando en realidad no es más que una construcción sociocultural que no tiene que ver con el aspecto biológico, sino más bien con el ideológico (Bodoque 20012, Beck-Gernsheim 2003, Sau 1994). A la mujer se le asignan, en función de un criterio biológico, tareas de reproducción natural y cuidado de la progenie, lo que la relega a un ámbito domestico bajo el dominio y dependencia del varón, quien se auto-adjudica la razón, la cultura, el ámbito público y político.

El predominio de la razón frente a lo natural permitió al varón dominar la organización social de acuerdo con sus valores, favoreciendo su supremacía frente a la mujer en beneficio propio, manipulando políticamente la regulación al acceso reproductivo y sexual de las mujeres. Se legitima una dominación a nivel de estado creando leyes que regulan la conducta social, estableciendo lazos de parentesco mediante el intercambio de mujeres y haciendo políticas en perjuicio de las mismas. El control de las mujeres se llevaba a cabo con una herramienta todavía vigente en la actualidad: el patriarcado. Su representación básica es la entidad familiar con estructura piramidal y jerárquica (Sau, 1994), en donde la mujer está situada en la base, ejerciendo el varón dominio no sólo sobre las mujeres, sino también sobre los varones más jóvenes. Los padres controlan el acceso de otros varones a sus mujeres mediante un contrato sexual, pacto llevado a cabo entre varones que tiene 
como fin disponer del cuerpo de la mujer visto como objeto reproductor (López Moratalla, 2011; Amorós, 2000). Para Rousseau el contrato sexual es un pacto social entre mujeres y varones, mediante el cual la mujer acepta el estado de subordinación al varón a cambio de protección y alimento (Cono ,1996).

La mujer, considerada inferior, se sitúa en un espacio privado y domestico dedicada al cuidado de la familia conforme a los estándares de supremacía masculinos, encubiertos por los valores morales destinados a perpetuar el dominio del varón sobre la mujer y transmitiéndolos de madres a hijas dentro del aprendizaje básico social (Amorós, 2000). Es la violencia simbólica que describe Bourdieu, la falta de conciencia por parte de las mujeres en cuanto a la dominación que sufren es lo que permite la continuidad del sistema (Bordieu, 2000). Todo ello apoyado sobre la pedagogía de que el fin de toda mujer es el matrimonio (reflejo social del contrato sexual) y la maternidad. La familia patriarcal es por tanto un modelo de sociabilidad definido como ideal por Rousseau, que distinguía perfectamente entre roles de géneros, encasillando a la mujer dentro de un ambiente reducido y dominado.

El reconocimiento de la igualdad y de los derechos de las mujeres es una clara amenaza para el orden patriarcal, pero lejos de desaparecer se ha producido un proceso de adaptación del mismo, evolucionando de un patriarcado de coerción a uno de consentimiento (De Grado, 2011). Hoy en día se admite la igualdad formal entre los dos sexos, aunque el discurso mantiene sutilmente el mismo contenido de falsa libertad, perpetuando la dominación masculina camuflada de igualdad (Camps, 2000; Díaz, 2003).

Frente al modelo de familia tradicional cuyo eje central era el matrimonio heterosexual está surgiendo una amplia variedad de estructuras familiares que conllevan una adaptación de los roles de género. El reconocimiento legal del matrimonio homosexual, así como al cambio situacional de la mujer en el seno familiar son dos de las causas fundamentales de la transformación familiar. El perfil actual femenino es una mujer trabajadora, independientemente de su nivel de formación, no relegada obligatoriamente al ambiente domestico. El tipo de empleo que ocupan las mujeres es en su mayoría a tiempo parcial. Según la Encuesta de Población Activa (EPA) del 2010 acerca de la conciliación de la vida familiar, el 97,3\% de las personas empleadas a tiempo parciales eran mujeres, las cuales elegían este tipo de contrato para hacerse cargo del cuidado de los hijos menores de 14 años o de otros parientes adultos o discapacitados (15,9\%). El precio elevado de estos servicios no está al alcance de las familias, obligando a la mujer a asumirlos en la mayoría de los casos. Un dato que lo confirma es la baja tasa de empleo femenino cuando incrementa el número de hijos menores de 12 años (Cigarini, 2006), justo al contrario que los varones, en donde el porcentaje aumenta. Para las mujeres de 25 a 49 años sin hijos la tasa de empleo en el año 2011 era de $68,1 \%$ y se reduce a $60,0 \%$ en el caso de tener hijos menores de 12 años. Con un hijo menor de 12 años el valor de la tasa es de $61,7 \%$ y de $58,9 \%$ en el caso de dos hijos menores de 12 años. Con tres hijos o más, el valor de la tasa es 47,6\%.(Díaz ,2003) La maternidad hace disminuir la tasa de empleo femenino.

La situación actual de la mujer es consecuencia de una evolución sociocultural lenta en el tiempo. Las primeras legislaciones europeas a favor de la maternidad y la infancia datan de la segunda mitad del siglo XX. En España la mujer trabajaba junto al marido en el campo, pero no es hasta la revolución industrial cuando se inicia el proceso regulador legal (Díaz, 2005). El código civil de 1851 permitía el acceso de la mujer al trabajo con expreso permiso paterno o marital, considerando el salario como bien ganancial. La ley Dato de 1900 reconoció un descanso por maternidad de tres semanas después del parto, con mantenimiento del puesto de trabajo sin remuneración económica. La mujer tenía prohibido, junto a los niños, desempeñar puestos en el sector de la minería o con peligros obvios, debiendo descansar los domingos. Todas estas medidas, más que proteger el derecho de las mujeres, estaban destinadas a fomentar el empleo de varones, ya que las mujeres al recibir menos salarios eran más contratadas en determinados sectores de la industria como el textil 
(Nielfa, 2003). Con la Constitución española de 1978 se reconoce la igualdad en derechos y deberes sin discriminación de sexo. A partir de entonces se fueron elaborando una serie de leyes que permitieran la conciliación de la vida laboral y familiar, así como medidas contra la violencia de género ( Orden PRE/525/2005, de 7 de Marzo), garantizar un acceso por igual a los puestos de la administración del estado (Orden APU/526/2005, de 7 de Marzo), activar políticas de real igualdad( Ley Orgánica 3/2007, de 22 de Marzo). Mediante el Plan Estratégico de Igualdad de oportunidades 2008-2011 se redefine el modelo de ciudadanía, el empoderamiento de las mujeres, la transversalidad de la perspectiva de género así como la innovación científica y tecnológica. Dentro de los planes estratégicos de desarrollo europeo para el 2020 se fomentan políticas de empleo destinadas a favorecer la igualdad del reparto de tareas domesticas entre ambos géneros, igualando las medidas de conciliación familiar, e incentivando a la mujer dentro del mundo profesional. Según la encuesta de la fuerza del trabajo de Eurostat, la diferencia en la tasa de empleo entre varones y mujeres es de $12.1 \%$ en España, observando que a menor formación mayor es la diferencia. En este caso la tasa europea es superior en UE27, $12.7 \%$.

La mujer dispone de menos tiempo de ocio, siendo la principal cuidadora de los hijos y demás miembros familiares, al mismo tiempo que reclama una mayor implicación del varón. Continúa ejerciendo su función de cohesión intergeneracional, debido a su papel fundamental en las relaciones personales que fomenta en la familia, mediante la satisfacción emocional de otros miembros familiares. Este dato demuestra la vigencia de la ética del cuidado propuesta por Gilligan, quien defiende como fortaleza esa capacidad de cuidado de los demás. En relación a las tareas domesticas ya no son exclusivas de la mujer, a pesar que ellas dedican una 3,46h al día a las labores del hogar frente 2,21 h que dedican los varones ${ }^{1}$.

Ante la salida de la mujer del ambiente domestico el patriarcado de consentimiento intenta mantener la prevalencia masculina sobre el hecho biológico de la gestación y el alumbramiento. La idea de la maternidad como único fin de toda mujer cambia a una maternidad de libre elección a pesar del condicionamiento sociocultural (Camps, 2000). Existen secuelas vigentes de la coerción del patriarcado tradicional que están presentes en las estructuras sociales, sobre todo mediterráneas, impregnando las estructuras cognitivas de las personas de ambos sexos a través de la cultura y la educación (Bordieu, 2000). Además se fomenta la exigencia de cumplir unos estándares de calidad que hacen que la mujer sea buena madre. Esta idea viene avalada por los avances del conocimiento que reconocen la importancia de la infancia para un correcto desarrollo de la persona adjudicando a la madre un papel relevante (Frischmuth ,2000).

\section{Maternidad}

La maternidad no es un simple proceso de reproducción biológica enmarcada en un cuerpo físico, sino que conlleva una construcción sociocultural propia de cada cultura. Es un hecho biopsicosocial (Sánchez B,1996).

El concepto tradicional de maternidad es una construcción cultural masculina para ejercer el control de la mujer presentándolo como único objetivo vital femenino (Branciforte, 2009; Otegui 1999). Dicho concepto en Occidente está influenciado por ideas griegas y judeocristianas, a las que posteriormente se han ido incorporando aspectos económicos capitalistas. Los griegos culpabilizaban la infertilidad en forma de arpías o gorgonas. Aristóteles consideraba a la mujer como un simple contenedor de semilla masculina, idea vigente hasta el descubrimiento del ovulo en el siglo XIX, a pesar que el aparato reproductor masculino ya se conocía en el siglo XVII (Bosch, 1999). El catolicismo consagra la maternidad con la imagen de la virgen, madre de dios inmersa en ideas de abnegación, amor incondicional y sufrimiento. La influencia capitalista va dirigida hacia una mujer blanca,

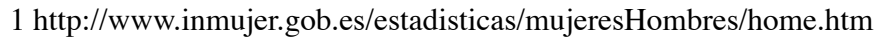


cristiana, con la función, no sólo, de procrear sino de fabricar la mayoría de los productos de consumo diario. En el siglo XIX, el siglo de las madres, se fomentó políticamente su papel, responsabilizándolas del aumento demográfico y de la mejora de la especie en el caso de regímenes totalitarios (Sánchez, 1996; Branciforte, 2009).

El patriarcado genera un discurso dominante de la maternidad con refuerzos sociales, políticos, económicos e incluso militares, atribuyendo a las madres una sabiduría generalizada por el propio hecho de ser madres. A medida que se aumenta el conocimiento científico y experto, en el pasado siglo XX, se ha ido devaluando la sabiduría cotidiana de las madres, sin embargo su importancia sigue vigente a través de la idea de realización de la mujer.

La infertilidad continúa teniendo un impacto social relevante afectando a las relaciones individuales de la mujer ya que la construcción del concepto de feminidad sigue basándose en los órganos sexuales y su óptimo funcionamiento (Campos 2011). La visión social de la mujer sin descendencia sigue siendo objeto de tristeza e infortunio, y criticado socialmente en algunos casos como egoísmo de la mujer.

La maternidad sigue representándose culturalmente como el eje central de toda mujer, el elemento común universal que las define a todas, e incluso surge el concepto de madre biológica con una importancia relevante (Livi 2012). La influencia social sobre la mujer para que sea madre se camufla bajo una aparente libertad de decisión. Ser padres se ha convertido en objeto de planificación (Beck-Gernsheim ,2003), dando lugar a la llamada maternidad/paternidad responsable. Se trata de una decisión vital, que debe tener en cuenta criterios no solo personales sino también económicos y profesionales. La decisión libre e individual de la mujer a disfrutar de su capacidad de gestación está limitada por distintos factores. Entre todos ellos emerge un condicionante físico conceptualizado como el reloj biológico. Esta idea surge en los años 70 del pasado siglo, con la pretensión de crear un conflicto en la mujer ante la decisión de ser madre o ejercer una profesión (Campos 2011).

Es un concepto discutido desde las distintas corrientes feministas, ya que lo consideran una reminiscencia del dominio masculino que pretende reavivar la condición natural de la mujer para la reproducción. Desde la ciencia producida a partir de los valores hegemónicos masculinos, es indiscutible que la capacidad de tener hijos no es igual en todas las etapas de la vida para ambos progenitores. Sin embargo la predominancia que se da en este aspecto al papel de la mujer sí que parece indicar que todo está orientado a crear de un sentimiento de angustia y culpabilidad en quien decide posponer la maternidad en beneficio de planes de vida individuales. Por otro lado se tiende a realizar una clasificación social a partir del ciclo vital en base al estado biológico: infancia/niñez, juventud/esposa-madre, madurez/abuela. De ello se espera socialmente distintos roles. Se trata de un modelo científico medico que se refleja en la ordenación social, adjudicando a la mujer diferentes funciones sociales dependiendo de la etapa biológica en que se encuentre (Bodoque, 2001). El reloj biológico pretendería convencer acerca del condicionamiento que la edad provoca en las condiciones biológicas de la reproducción, por lo tanto si desea tener hijos, debe ser cuando se es joven.

Desde un punto de vista feminista se trata una adaptación de la idea tradicional de que la mujer debe sentir la llamada de la naturaleza en su fin primordial de procrear, el binomio de mujer-naturaleza que ha perdurado hasta nuestros días (De Grado, 2011). Las mujeres disponen de una etapa fértil que va desde la menarquía hasta el climaterio, con gran variabilidad de los 12 a los 50 años. Durante la misma su capacidad reproductora varía con los años, disminuyendo progresivamente el número de folículos ováricos y de la calidad de los ovocitos, lo que afecta su expresión genética. Un grupo determinado de genes relacionados con la regulación del ciclo celular se expresan de forma distinta en mujeres menores de 35años que en las mujeres de 37-39 años (Livi, 2012). En los varones a partir de los 35 años también disminuye la fertilidad, sin embargo, este hecho no es divulgado socialmente. La mayoría de la gente considera que un varón puede procrear indefinidamente, dando notoriedad pública al varón que es padre a una edad avanzada. 


\section{Mujer contemporánea}

Las sociedades actuales tienen como característica fundamental el proceso de cambio rápido y constante que genera un sentimiento de riesgo al que se enfrentan las personas por medio de la planificación. La preocupación por el futuro incierto obliga a la previsión de todas las decisiones vitales. Esta perspectiva condiciona la vivencia de la maternidad en la mujer. La decisión de no tener hijos puede interpretarse como postura de evitar riesgos de la misma forma que la mujer que triunfa a nivel profesional suele ser vista como perdedora en el ámbito domestico y viceversa. Es la manifestación de la dominación social masculina vigente (Beck-Gernsheim 2003; Bordieu, 2000). En el mundo occidental se fomentan estilos de vida que rinden culto al cuerpo, a la salud física, a la belleza y a la juventud, aspectos que se ven claramente afectados durante el proceso de gestación. Se produce una paradoja en la decisión de ser o no madre, ya que se contrapone la pérdida de juventud frente a una maternidad responsable y planificada, con estabilidad laboral y económica, priorizando el bienestar (Sánchez, 1996; Branciforte, 2009).

El cambio social respecto a la mujer es un fenómeno muy reciente. En España, en siglo XIX y principios del XX, solo se admitía el derecho de las mujeres a saber leer y escribir, fomentando la dedicación de la mujer al ámbito domestico, permitiéndolas el acceso a profesiones consideradas femeninas como institutriz o maestra (Delgado, 1993; Maroto, 2004). Estas profesiones disponían de una estructura patriarcal donde se reflejaba la situación social de la mujer. Hoy en día la mujer tiene una formación académica y obtiene incluso mejores resultados que los varones. La educación obligatoria permite el acceso de todas las niñas en igualdad de condiciones que los varones. El abandono temprano de la escuela es mayor en varones que en mujeres (31\% frente a $21.9 \%)$. En el ámbito universitario un $57.95 \%$ de los estudiantes universitarios que terminan la carrera son mujeres. Los doctorados son prioritariamente masculinos, pero dentro de las mujeres que se doctoran, la mayoría lo hacen en franjas de edad de 20-30 años, mientras que los hombres lo hacen más mayores. La mujer decide formarse antes y más rápido que los varones ya que es conocedora de que tras la maternidad las dificultades de formación aumentan.

En el sistema neoliberal la competitividad humana es el motor de la sociedad. La no igualdad en salarios además de la ausencia de legislaciones que favorezcan el proceso de maternidad, hace que esta se posponga hasta disponer de una estabilidad económica y profesional (Beck-Gernsheim 2003). No hay que olvidar el mito del amor romántico, vestigio de un patriarcado de coerción que fomenta la idea de necesidad de un hombre para formar una familia, haciendo a la mujer dependiente de su amor (Verdú, 2014). Las mujeres planifican ser madres en un determinado momento de su vida, posterior a la obtención de metas laborales o formativas. Este retraso de la maternidad conlleva un envejecimiento de la mujer en relación a su capacidad reproductiva, lo que puede generar problemas de concepción. Cuando se puede no se quiere y cuando se quiere, no se puede.

El surgimiento de Técnicas de Reproducción Asistida (TRA) ha permitido a disponer de una alternativa a los problemas en la concepción. Sin embargo, autores como Mercedes de Grado opinan que las TRA continúan fomentando el rol de la mujer como madre, perpetuando su posición en el patriarcado (De Grado, 2011). No hay que olvidar que estas técnicas son llevadas a cabo en su mayor parte por empresas privadas en busca de beneficios económicos, lo que podría restar objetividad a los datos que ofrecen en relación a la fertilidad de la mujer. Se está produciendo una mercantilización de la maternidad, no solo en base a las TRA, sino también con otras formas de maternidad (vientres de alquiler, adopciones o donaciones de óvulos y esperma). La posibilidad de acceder a la maternidad con recursos económicos podría generar un cambio conceptual de la maternidad, facilitando la decisión a aquellas mujeres con mejores recursos y obligando a las mujeres en peor situación económica a una decisión maternal más temprana 


\section{Conclusiones}

Es un hecho demostrado que se ha producido un aumento en la edad de la mujer para la maternidad. Sin embargo en el caso del varón no se encuentran datos específicos sobre la edad media de la paternidad, o niveles de fertilidad según la edad. Esto corroboraría la idea que todavía se centra la maternidad en la mujer, adjudicándole mayor importancia a la parte femenina de la reproducción, cuando el varón, posiblemente tiene los mismos problemas. Ambos tienen un condicionamiento biológico pero culturalmente se sigue dando más importancia al de la mujer. Se debe resaltar la facilidad en la recolección de datos femeninos ya que la maternidad implica un proceso hospitalario que obliga a la obtención de información específica de carácter sanitario respecto a la madre, que posteriormente pueden ser extrapolables a otros fines estadísticos.

El aumento de la infertilidad es un dato de difícil interpretación, ya que por un lado la cifra de $1 \%$ en los últimos veinte años, no parece muy alarmante, y más teniendo en cuenta que la esperanza de vida de los países occidentales ha ido en aumento. Nacen menos pero se vive más. La crítica desde el feminismo resalta una manipulación económica en intereses de las técnicas de reproducción. Esta idea manifiesta la duda de una ciencia hecha desde el dominio masculino. Sin embargo se debe evitar caer en los mismos errores patriarcales desde el punto de vista femenino.

La organización social basada en el patriarcado y sustentado por ideologías ya sean filosóficas (Rousseau) o religiosas (catolicismo) está obsoleta. En el patriarcado el eje central era el matrimonio heterosexual sobre el que se construía los roles de género. En matrimonios homosexuales la condición de género en la pareja no existe, no se predetermina ningún rol específico sino que debe construirse un modelo individualizado de los mismos. Este punto demuestra abiertamente que la desigualdad de sexos y la construcción de género es completamente sociocultural. En la época de transición actual, la pluralidad social exige distintos modelos familiares, con una readaptación de los roles de género e incluso una nueva definición del propio concepto de género. La variabilidad familiar actual coloca a las sociedades ante un futuro incierto, pero las dota con la esperanza de hacerlas más igualitarias en relación al género. Frente a las opiniones que defienden el modelo familiar tradicional como elemento imprescindible para la educación de los hijos, algunos estudios acerca de los distintos modelos familiares, avalan que el desarrollo de los niños no se ve afectado, permitiendo que futuras generaciones se formen dentro de unos marcos conceptuales más amplios e igualitarios (Oliva, 2014).

La mujer en igualdad de derechos y deberes desde la legalidad de los sistemas democráticos, va transformando el ámbito domestico. El varón se incorpora progresivamente a las tareas domesticas y el cuidado de los hijos. La existencia de planes estratégicos por parte de los gobiernos europeos para fomentar la incorporación de la mujer al ámbito público, deja la puerta abierta a la completa inmersión de la mujer en la sociedad, en la política y en definitiva en la construcción sociocultural.

La maternidad tardía es una manifestación reminiscente del patriarcado. La incorporación de la mujer a un mundo hecho por y para hombres, donde no existen condiciones laborales que favorezcan la maternidad y conciliación de la vida familiar, hace que la mujer posponga la maternidad hasta poder disponer de condiciones óptimas para ello. Se ofrece la maternidad como una libre elección de la mujer, apoyado por los nuevos modelos de familia y por la tecnología moderna a través de las TRA (Branciforte, 2009). Sin embargo, se debe reconstruir culturalmente el concepto de paternidad-maternidad desde un origen de libre decisión, con la carga de responsabilidad conjunta que incluya reparto de tareas de forma equilibrada, sin más sobrecargas por el mero hecho de ser padre o madre, sin entrar en la influencia del lenguaje que de por sí ya establece diferencias. De la misma manera que se produjo un gran debate social con el término matrimonio para parejas homosexuales, 
también debería producirse una re-denominación de los conceptos de padre y madre, mas aun teniendo en cuenta la multiplicad de conceptos en relación a la maternidad que están surgiendo en función de las distintas circunstancias que la rodean: maternidad biológica, embarazos de alquiler, adopción, fecundaciones in vitro.

Debe evitarse caer en el error de adjudicar la capacidad reproductora como decisión unilateral de la mujer, sin tener en cuenta que la opinión del varón es fundamental y también está sujeta a presiones socioculturales.

\section{Biblografía}

Amorós Puente, Celia (2000). Sobre el feminismo, proyecto ilustrado y postmodernidad. Madrid, Ed. Cátedra.

Beck-Gernsheim, Elisabeth (2003). La reinvención de la familia. En busca de nuevas formas de convivencia. Barcelona, Ed. Paidos.

Bodoque Puerta, Yolanda (2001). "Tiempo biológico y tiempo social. Aproximación del ciclo de las mujeres”, Gaceta de Antropología, 1, art.12

Bourdieu, Pierre (2000). La dominación masculina. Barcelona, Ed. Anagrama.

Bosch Margarita, Ferrer, V, y Gili, M. (1999). Historia de la misoginia. Barcelona, Ed. Anthropos.

Branciforte, Laura (2009). "La maternidad: el tránsito desde la tradición a la elección en la edad contemporánea". Cuadernos Kóre. Revista de Historia y Pensamiento de Género, 1, 1: 41-52.

Camps Cervera, Victoria( 2000). El siglo de las mujeres. Madrid, Ed. Cátedra.

Campos Mansilla, Beatriz (2011). "La falta de descendencia biológica. Una lectura social y feminista de la infertilidad de las mujeres", Cuadernos Kóre. Revista de Historia y Pensamiento de Género, 1, 4: 97-121.

Cigarini, Ua (2006). "El doble SI de las mujeres a la maternidad y al empleo", DUODA: Estudis de la diferencia sexual, 30: 51-59.

Cobo Rosa ( 1996),"Sociedad, democracia y patriarcado en Jean Jacques Rousseau". Papers, 50 265-280.

Delgado, Margarita (1993).“Cambios recientes en el proceso de formación de la familia”, Reis: Revista española de investigaciones sociológicas, 64: 123-154.

De Grado Gónzalez, Mercedes (2011). "Semen, óvulos y úteros nómadas. Representaciones sobre mujer, maternidad y nuevas técnicas de reproducción asistida", Icono 14, 1,(9):161-174.

Díaz Mintegui, Carmen (2003). “Tradiciones culturales y legitimación del poder masculino”, Gazeta de Antropología, 19, 15: 1-19.

Díaz Fernández, Paloma (2005). "La dictadura de Primo de Rivera: una oportunidad para la mujer”. Espacio Tiempo y Forma. Serie V, Historia Contemporánea, 17.

Frischmuth, Silke (1993). "La maternidad en el pensamiento feminista occidental", Revista Artículos y Ensayos de Sociología Rural, 7: 45-56.

Instituto nacional de la mujer. Mujeres y Hombres 2013. Extraído el 23 Abril 2014 desde http://www. inmujer.gob.es/estadisticas/mujeresHombres/home.htm

Livi Bacci Massimo, (2012). "Low fertility in historical perspective". Population and Development Review, 38: 72-82.

López Moratalla, N; Palacios Ortega, S; Lago Fernández, M; y Beunza Santolaria, M. (2011).“Retraso en la edad de procreación e infertilidad. El recurso de la reproducción asistida y selección de embriones. El problema intergeneracional". Cuadernos de bioética XXI, 2: 325-340

Maroto Navarro, Gracia; García Calvente, M, y Mateo Rodríguez, I ( 2004) "El reto de la maternidad en España: dificultades sociales y sanitarias". Gaceta sanitaria, 18, 13-23.

Nielfa Cristobal, Gloria (2003). "Trabajo, legislación y genero en la España contemporánea: los orígenes de la legislación laboral”. Gálvez Muñoz, Lina; Sarasúa, Carmen (Coord.). ¿Privilegios o eficiencia?: mujeres y hombres en los mercados de trabajo: 39-56. Alicante: Universidad de Alicante

Oliva, A ; Arranza, E.; Parra, A., y Olabarrieta, F. (2014). "Family structure and children adjustement in Spain". J. Child Fam. Studies, 23 10-19. 
Otegui, Rosario (1999). "La construcción social de las masculinidades". Rev. Política y sociedad, 32: $151-160$

Sánchez Bringas, Ángeles (1996). “Cultura patriarcal o cultura de mujeres: Una reflexión sobre las interpretaciones actuales". Política y Cultura, 6: 161-168.

Sau, Victoria (1993). La maternidad: una impostura, DUODA: Estudis de diferencia de sexual, 6: 97-113

Verdú Delgado, Ana Dolores (2014). "El amor en la sociedad de consumo", Gazeta de Antropologia, 30: $1-14$.

\section{3}

